\title{
Zika and chikungunya infections in Brazil: reviewing the epidemic and treatment options
}

\author{
Danillo Lucas Alves Esposito ${ }^{[1]}$ and Benedito Antônio Lopes da Fonseca ${ }^{[1]}$
}

[1]. Departamento de Clínica Médica, Faculdade de Medicina de Ribeirão Preto, Universidade de São Paulo, Ribeirão Preto, São Paulo, Brasil.

Arboviruses (arthropod-borne viruses) are transmitted by infected arthropods to susceptible individuals, causing a variety of diseases in humans. The most common infections in humans caused by mosquito-borne arboviruses are dengue, Zika and chikungunya, all of them primarily transmitted through the bite of an infected female mosquito from the Aedes genus.

In addition to the dengue epidemic, Brazil is currently facing two new epidemics caused by arboviruses. One is caused by the chikungunya virus. The epicenter of the outbreak is located in the Northeast region of the country, but it is moving west towards the central region of Brazil. The other is caused by the Zika virus, which was also initially detected in the northeast but has spread to all regions of the country. According to the Brazilian Ministry of Health, almost 230,000 suspected cases of chikungunya infection were notified in 2015 and 2016, and 200,000 cases of Zika infection have been reported since $2014^{(1)}$. These numbers are based on clinical/epidemiological criteria, since there are no commercial laboratory tests that are sensitive enough to confirm these infections, especially of Zika.

Zika virus belongs to the Flaviviridae family. It was isolated in 1947 from an infected Rhesus monkey used as a sentinel animal for yellow fever research in $\mathrm{Uganda}^{(2)}$. It has been reported that the disease is usually asymptomatic, with an estimated $20 \%$ of infected individuals developing a mild dengue-like disease with low-grade fever, maculopapular pruritic rash, myalgia, arthralgia, and non-purulent conjunctivitis ${ }^{(3)}$.

Chikungunya virus is an alphavirus that was first isolated in Tanzania in 1950 and reached the Americas in 2013, initially arriving in the Caribbean islands and then spreading to other countries in Latin and South America. In 2014, a huge epidemic broke out in Feira de Santana, Bahia State, Brazil ${ }^{(4)}$, and since then, an outbreak of chikungunya has been ongoing in the northeast region of the country. In contrast to Zika virus infections, chikungunya virus infections are usually followed

\footnotetext{
Corresponding author: Dr. Benedito Antônio Lopes da Fonseca. e-mail: baldfons@fmrp.usp.br

Received 10 October 2016

Accepted 13 October 2016
}

by a high rate of symptomatic disease, with intense myalgia, high fever $\left(>39^{\circ} \mathrm{C}\right)$ that appears in an early stage of the disease, and a characteristic arthralgia that, in some cases, can persist for months or years.

Detection of chikungunya and Zika virus viremia is the preferred and most specific method of diagnosis, but due to the absence of a nationwide infrastructure to detect these viruses by molecular methods and to the costs involved with these techniques, not all individuals can be diagnosed during the acute disease phase. Thus, diagnosis relies on serology and in particular on Immunoglobulin M (IgM) antibody detection, as IgM antibodies are produced within a few days after contact with a pathogen. Immunoglobulin $\mathrm{G}$ (IgG) antibodies are associated with lifetime protection, thanks to a more specific interaction with the pathogen, but they are not adequate for diagnosis due to the need for both acute and convalescent samples to reach a specific diagnosis ${ }^{(5)}$. To make things worse, because of their phylogenetic relationship, cross-reactivity can occur between closely related viruses, such as dengue and Zika or chikungunya and Mayaro viruses, all of which circulate in the Brazilian territory. Several studies have shown cross-reactivity between dengue and Zika virus antibodies $^{(6)}$, and, as noted in one of the articles published in the current issue of this journal, these findings suggest that at least some of the large number of dengue cases reported in 20152016 in Brazil could, in fact, have been Zika virus infections.

In addition to the lack of specific laboratory tests, since these infections are relatively new to the country, most physicians are still learning how to differentiate one disease from another and how to treat each one of them properly.

In this issue of the Journal of the Brazilian Society of Tropical Medicine, two articles will highlight and discuss some of the problems associated with these new infections in Brazil. The first article, entitled Zika in Pernambuco: rewriting the outbreak, describes a clinical-epidemiological retrospective study on previously notified dengue-like diseases and discusses the importance of the correct diagnosis of a disease when there is more than one circulating arbovirus in a specific region at the same time. In this sense, an adequate discussion is provided against the approach taken by the Ministry of Health and the delay in recognizing that a surveillance system for Zika should have been implemented earlier. In a large cross-sectional 
study, the authors conclude that the highest number of cases attended in a reference hospital in Pernambuco state was due to Zika and not to dengue. Although the data in this study deserve a careful analysis, diagnoses were also unfortunately based on the symptoms presented by the patients, which might have inflated the number of Zika cases. However, since there is no suitable diagnostic method for Zika virus infections, their approach was interesting and confirmed the common knowledge already acquired by the Brazilian Ministry of Health.

Similar to treatments available for dengue and Zika virus infections, treatment for chikungunya virus infections is directed only to the symptoms, and since joint involvement can become chronic and sometimes incapacitating, it is very important to provide the proper treatment to individuals, especially when aiming to provide a better quality of life. The review entitled Pharmacological pain treatment in Chikungunya: a guideline describes all possible treatment options available to alleviate the symptoms of chikungunya virus infection according to age and pain intensity. The approach described there, although addressing only the most common painkillers available and not suggesting new strategies to control disease progression, is a useful guideline to be followed by the general practitioner treating a patient with chronic pain resulting from chikungunya virus infection. The article provides several well-elaborated flowcharts and questionnaires to be used according to disease presentation that, hopefully, will help diminish the pain associated with the severe joint and muscle involvement of the disease.

Several promising diagnostic techniques to differentiate these infections are currently being developed and undergoing careful evaluation, and, if successful, with help to establish a good clinical profile and correct characterization of each disease. Since these diagnostic techniques are not yet available, a thorough study of clinical manifestations of these diseases will help to define parameters that may differentiate one from the other, and while there is no vaccine or medication to prevent the progression of chikungunya joint disease, guidelines such as the one described in this issue of the Journal of the Brazilian Society of Tropical Medicine will help physicians provide the best possible care to patients infected with the chikungunya virus.

\section{Conflict of interest}

The authors declare that there is no conflict of interest.

\section{REFERENCES}

1. Ministério da Saúde. Secretaria de Vigilância em Saúde. Boletim Epidemiológico, volume 47, $\mathrm{n}^{\circ} 33$, 2016. Disponível em: http:// portalsaude.saude.gov.br/images/pdf/2016/setembro/16/2016-028--Dengue-SE32.pdf. Acesso em 23 set 2016.

2. Simpson DI. Zika Virus Infection in Man. Trans R Soc Trop Med Hyg 1964; 58:335-338. doi: 10.1016/0035-9203(64)90201-9.

3. Hennessey M, Fischer M, Staples JE. Zika virus spreads to new areas - region of the Americas, May 2015-January 2016. MMWR Morb Mortal Wkly Rep 2016; 65:55-58.

4. Nunes MRT, Faria NR, de Vasconcelos JM, Golding N, Kraemer MU, de Oliveira LF, et al. Emergence and potential for spread of Chikungunya virus in Brazil. BMC Med 2015; 13:102. doi: 10.1186/ s12916-015-0348-x.

5. Charrel RN. Diagnosis of arboviral infections - A quagmire of cross reactions and complexities. Travel Med Infect Dis 2016; 14:11-12. doi: 10.1016/j.tmaid.2016.01.006.

6. Dejnirattisai W, Supasa P, Wongwiwat W, Rouvinski A, BarbaSpaeth G, Duangchinda T, et al. Dengue virus sero-cross-reactivity drives antibody-dependent enhancement of infection with zika virus. Nat Immunol 2016; 17:1102-1108. 